\title{
Introduction to the Judgement, Big Data-Analytics and Decision-making Minitrack
}

\author{
David Pauleen \\ Massey University \\ d.pauleen@massey.ac.nz \\ Kasuni Weerasinghe \\ Massey University \\ w.m.k.g.weerasinghe@massey.ac.nz \\ Nazim Taskin \\ Massey University \\ N.Taskin@m assey.ac.nz \\ Ali Intezari \\ University of Queensland \\ a.intezari@business.uq.edu.au
}

\begin{abstract}
2021 is the first year that the Judgement, Big DataAnalytics and Decision-making mini-track has been offered. The track's objective is to monitor and advance our knowledge of the convergent technologies of Big Data and analytics and their role in augmenting knowledge for better management decision-making. The track attracted seven submissions of which five were accepted. The papers form a diverse group, offering case studies of big data analytics projects and critical analysis of various factors that impact the successful or unsuccessful use of data/analytics in organizational settings.
\end{abstract}

\section{Introduction}

The goal of the Judgement, Big Data-Analytics and Decision-making mini-track is to monitor and advance our knowledge of the convergent technologies of Big Data and analytics and their role in augmenting knowledge for better management decision-making. The emphasis of the track is on the humanistic aspects of knowledge innovation, by investigating how dataanalytics, and big data analytics in particular, can be used to support human judgement and organizational knowledge.

Gathering, analyzing and judging reliable data and information, and more importantly, transforming them into actionable knowledge is becoming increasingly critical in decision-making (Intezari \& Gressel, 2017; Nicolas, 2004; Abedin et al, 2018). Human judgement, based on experience and knowledge, plays an important role in all facets of decision-making, but may be incomplete in certain decision situations. The study of the transformation of data into useful information and eventually knowledge and wisdom through the use of increasingly sophisticated analytics for management decision making is an important, emerging field of research (Pauleen \& Wang, 2017) and deserves further study.

The papers accepted in this track form a diverse group, offering case studies of big data analytics projects and critical analysis of various factors that impact the successful or unsuccessful use of data/analytics in organizational settings.

\section{Summary of the Papers}

1. Practical Wisdom and Big Data Dilemmas: The Case of the Swedish Transport Administration Authors: Lena Hylving \& Susanne Lindberg

Using big data in organizations has the potential to improve innovation, accuracy, and efficiency. Big data is also connected with risks for both the organization and society at large. It is therefore important to improve our understanding of potential consequences of implementing and using big data. This research studied the Swedish Transport Administration to understand their attitude towards implementing big data for prediction of, for example, the need for road maintenance. The analysis identified four moral dilemmas that the organization deals with in connection to big data. These these dilemmas are discussed from the perspective of practical wisdom. Practical wisdom is manifested in context-dependent actions connected to open-mindedness, reflection and judgment. It can be summed up as "the reasonable thing to do" in a unique situation where "not-knowing" is a helpful resource when making wise decisions. This paper seeks to shed light on the importance of practical wisdom when implementing big data. 
2. Cognitive biases in developing biased Artificial Intelligence recruitment system

Author: Melika Soleimani, A. Intezari, N, Taskin \& D.Pauleen

Artificial Intelligence (AI) in a business context is designed to provide organizations with valuable insight into decision-making and planning. Although AI can help managers make decisions, it may pose unprecedented issues, such as datasets and implicit biases built into algorithms. To assist managers with making unbiased effective decisions, AI needs to be unbiased too. Therefore, it is important to identify biases that may arise in the design and use of AI. One of the areas where AI is increasingly used is the Human Resources recruitment process. This article reports on the preliminary findings of an empirical study answering the question: how do cognitive biases arise in AI? A model determining people's role in developing AI recruitment systems is proposed Identifying the sources of cognitive biases can provide insight into how to develop unbiased AI. The academic and practical implications of the study are discussed.

3. Beyond the Hype: Why Do Data-Driven Projects Fail?

Authors: Tatiana Ermakova, Julia Blume, Benjamin Fabian, Elena Fomenko, Marcus Berlin \& Manfred Hauswirth

Despite substantial investments, data science has failed to deliver significant business value in many companies. So far, the reasons for this problem have not been explored systematically. This study tries to find possible explanations for this shortcoming and analyses the specific challenges in data-driven projects. To identify the reasons that make data-driven projects fall short of expectations, multiple rounds of qualitative semi-structured interviews with domain experts with different roles in data-driven projects were carried out. This was followed by a questionnaire surveying 112 experts with experience in data projects from eleven industries. The results show that the main reasons for failure in data-driven projects are (1) the lack of understanding of the business context and user needs, (2) low data quality, and (3) data access problems. It is interesting, that $54 \%$ of respondents see a conceptual gap between business strategies and the implementation of analytics solutions. Based on these results, recommendations are given for how to overcome this conceptual distance and carrying out data-driven projects more successfully in the future.

4. Factors Impacting the Influence of Analytic Capabilities on Organizational Performance in Higher Education

Authors: Cory Campbell, Philip Cola \& Kalle Lyytinen

In response to changing fiscal needs and opportunities, higher education institutions have adopted new ways to use financial information for improved decision making. Drawing upon resource based theory this paper examines the connection between university level data analytic capabilities and organizational performance. The paper posits this relationship to exist through a serially mediated path of data-driven culture and data quality. The study provides empirical evidence that establishing a data-driven culture contributes to data quality which together result in increased organizational performance. The serial mediation pathway creates a positive effect between data analytic capabilities on organizational performance. This is critical information relative to both resource based theories and practical implications for higher education relative to beginning the investment cycle at the organizational culture level related to use of data.

5. Investigating Insensitivity to Prior Probabilities in Merger and Acquisition (M\&A) Decision Making Authors: James McGaughan \& Shobha ChengalurSmith

This paper investigates the high failure rates of Mergers and Acquisitions (M\&As) over the last several decades, despite greater access to data, sophisticated business intelligence (BI) and data analytics (DA) tools, and work by industry professionals and academics to improve outcomes. The paper explores the possibility that the representativeness heuristic could play a role, and specifically, if prior probabilities are being ignored or discounted in M\&A evaluations. The hypothesis is confirmed using a regression discontinuity in time (RDiT) model and a two-way fixed effects model. Highlighting the negative consequences of this heuristic on management decisions may promote the use of datadriven decision making and the role of analytics in formulating business strategy. 